\title{
Risk of cardiovascular disease in people taking psychotropic medication: a literature review
}

Herbert Mwebe, Danielle Roberts

\begin{abstract}
In people with serious mental illness, mortality and morbidity are significantly increased compared to the general population. This is often as a result of physical health problems; about two-thirds of premature death in people with serious mental illness has been attributed to natural causes including cardiovascular disease and diabetes. Cardiovascular disease risk in people with serious mental illness is higher than the general population, this risk is further increased in those with serious mental illness taking psychotropic medication. In this article, cardiovascular disease risk in serious mental illness, particularly the association with psychotropic interventions and monitoring required, is examined.
\end{abstract}

Key words: serious mental illness; cardiovascular disease; monitoring; psychotropic medication.

\section{Introduction}

Cardiovascular disease is a general term for conditions that affect the heart or blood vessels of the circulatory system. The main types of cardiovascular disease include: coronary heart disease, aortic disease, peripheral artery disease, arrhythmias, valvular heart disease and cardiomyopathy. Coronary heart disease, also known as ischaemic heart disease, is one of the most prevalent chronic diseases. Coronary heart disease results from a complete or partial obstruction of arterial blood supply to the heart; often as a result of atherosclerosis - a build of fatty deposits in the coronary arteries.

Reduced blood supply to the heart muscle can cause chest pain and angina which, if left untreated, can lead to a heart attack (myocardial infarction). Diagnostic laboratory blood tests, radiological, electrophysiological investigations and a physical examination of associated symptoms (exertional chest pain, breathlessness, ankle swelling, leg pain) are conducted to formulate a comprehensive treatment plan. Additionally, screening should include enquiries about common cardiovascular disease risk factors including obesity, smoking, diabetes, hypertension, high cholesterol and physical inactivity when taking a full health history including family history, medical history, recreational drug use and psychosocial factors. Cardiovascular disease is closely linked to other long-term conditions including hypertension, diabetes, mental illness and chronic kidney disease, it is useful to offer additional screening in people presenting with signs and symptoms akin to these conditions to proactively identify cardiovascular disease risk early and offer necessary management (Lester et al, 2012; Tosh et al, 2014).

Despite the recent reduction in cardiovascular disease-related mortality in the UK general population (because of improved screening and monitoring interventions), cardiovascular disease remains a significant economic burden to the UK in terms of morbidity and costs (Bhatnagar et al, 2014). Cardiovascular disease-related deaths cost the UK economy (including disability, working days lost, premature death) approximately $£ 19$ billion each year (British Heart Foundation, 2018; Heart UK, 2018). According to the British Heart Foundation (2018), heart and circulatory complications associated with cardiovascular disease cause just over a quarter (26\%) of all deaths in the UK, accounting for 150000 deaths in a year, which is an average of 410 deaths each day. Cardiovascular disease is also a key cause of mortality and morbidity among people with diabetes. The American Diabetes Association reported 
that cardiovascular problems in people with either Type 1 or Type 2 diabetes were accountable for $27 \%$ of the total cost of treating and managing diabetes in the US (American Diabetes Association, 2013).

\section{Physical health needs (including cardiovascular disease risk) monitoring in severe mental illness}

Serious mental illness, such as schizophrenia, psychotic depression, bipolar, schizoaffective disorders, multi-diagnoses including learning disability and substance dependency, are debilitating and have complex life-limiting symptoms, including hallucinations, delusions, and social dysfunction. While increased suicide rates contribute to mortality in serious mental illness (Laursen et al, 2012), increased mortality in this patient group is from natural causes such as cardiovascular disease, diabetes, cancers, respiratory-related and complications (Holt, 2012; Mangurian et al, 2016). The impact of this reality is that life expectancy in people with serious mental illness is reduced by up to 25 years compared to the general population (Nash, 2013; Mutsatsa, 2015).

There is increased risk of adverse effects including weight gain, neurological, vascular, glycaemic and lipid abnormalities associated with the use of psychotropic medication. Cardiovascular disease risk is significantly increased further in patients where polypharmacy and high dose antipsychotic treatment regimens are evident, particularly when the drugs used have known iatrogenic adverse effects on the cardiovascular system. In addition, the impact of socioeconomic factors on the course of mental illness including social deprivation, poor access to health interventions, and poverty in people with serious mental illness further increases the risk of physical/medical problems (Mwebe, 2017, 2018). Correll et al (2017) conducted a large meta-analysis of cardiovascular disease prevalence in serious mental illness, which included 3.2 million patients and 113 million controls (people without mental illness sampled from the public), from 92 studies across 4 continents and 16 countries (including UK, USA, France, Australia, Sweden). The researchers found that the pooled cardiovascular disease prevalence in patients with serious mental illness was $10 \%$; while cardiovascular disease prevalence specifically in people with schizophrenia was $11 \%$, major depression was slightly higher at $11.7 \%$ and cardiovascular disease rates in people with bipolar disorder was $8.4 \%$. People with serious mental illness were found to have a significantly increased risk of developing cardiovascular disease over time and cardiovascular diseaserelated deaths compared to those without mental illness (Correll et al, 2017).

While strategies to improve physical health in people with serious mental illness have been developed and are now routinely evident in national and local health provider policies and frameworks (Shiers et al, 2014), the physical health needs of people with serious mental illness who are frequently in contact with health professionals and health services are not always promptly and adequately met (Hardy and White, 2013; Robson et al, 2013). A biopsychosocial approach to screening and management for both mental and physical health problems is enshrined within the care programme approach including care planning (Pilgrim, 2017). However, various challenges presenting at the individual, system and community level often hinder the successful development and delivery of effective care interventions in the management of physical and mental health care needs. These factors range from lack of funding and financial constraints on health service provision, inadequate IT/communication systems between provider services (different health care recording platforms in primary and secondary care), the severity and complexities of symptoms of mental illness, mental health stigma and social deprivation, lack of skills and knowledge and staff poor attitudes (Silverwood et al, 2019).

Mutsatsa (2015) argues that stigma ascribed to mental illness may explain why health professionals are more likely to attribute symptoms of physical ill health to mental illness, and this could possibly explain the several missed opportunities in offering timely interventions for people with serious mental illness 
presenting with physical/medical problems. A training needs analysis of mental health nurses' knowledge and skills of physical care practices in mental health settings found that nurses lacked confidence and up-to-date clinical knowledge and skills to tackle physical health complaints of patients (Nash, 2013; Mwebe, 2017).

At the time of writing, NHS health checks offer a person-centred approach to identify and screen for cardiovascular disease/other physical (medical) risk in individuals at high risk (hypertension, diabetes, stroke, overweight, heart attacks) of developing cardiovascular disease, including those over the age of 50, individuals with serious mental illness currently taking psychotropic medication, and those who may have a diagnosis of a serious mental illness (schizophrenia, bipolar disorder, psychotic depression) but are in remission and are not currently taking medication (NHS England, 2014a). Recommended annual physical monitoring checks in people with serious mental illness include advice on unhealthy lifestyle behaviours including smoking, recreational drug use, poor diet, poor oral hygiene, sedentary lifestyle and risky sexual behaviours. Blood tests, routine electrocardiogram and vital checks (blood pressure, pulse, weight monitoring) are recommended and should be offered before and after starting medications in serious mental illness.

In the UK, and globally, this practice gap disparity around physical health care, particularly screening and monitoring of cardiometabolic risk in people with serious mental illness has triggered a major shift in policy (Lester, 2012; Health Education England, 2016; NHS England, 2014a; Care Quality Commission, 2017; World Health Organization, 2018). Over the last decade, the focus has been around increasing and improving uptake of screening interventions both in primary and secondary health sectors and improving communication between services (inpatients, general practice and community settings). To do this, NHS Trusts and commissioners are investing in staff training and upskilling mental health staff to be ready and prepared to offer physical health assessments and monitoring. In some services, where secondary and primary patient care recording IT systems have been developed to share (communicate) some health informatics, this is a much welcome step to improving communication between provider services. To provide clear guidance and clarity to minimise confusion around roles and responsibilities for physical health monitoring, local policies informed by national levers and guidelines have been developed by provider/ commissioning services (NHS Trusts, Clinical Commissioning Groups).

\section{Cardiovascular disease risk in people of Asian and African-Caribbean background with serious mental illness}

Cardiovascular disease risk in people of Asian and African-Caribbean background is higher than in the general population (British Heart Foundation, 2018). Current guidelines recommend that patients from black and ethnic minority backgrounds who are at greater risk of cardiovascular disease should be offered screening at regular intervals depending on individual risk and at least 6-12 monthly checks. Incidentally, a mental health services dataset by NHS Digital (2018) found that among all ethnic groups, people from the black ethnic group were most likely to have been detained under the Mental Health Act 1983 than other groups. Further evidence also found that patients from African-Caribbean backgrounds are routinely prescribed higher doses of antipsychotic medication, especially typical antipsychotic medication compared with other ethnic groups (Copeland et al, 2003; Das-Munshi et al, 2018). Typical antipsychotics are especially associated with greater cardiovascular disease risk; for example, medications including haloperidol, phenothiazines, depot preparations and tricyclic antidepressants block alpha-1, alpha-2 adrenergic receptors and muscarinic receptors which can result in cardiovascular symptomatology (i.e. arrythmia, hypotension, dizziness). 


\section{Psychotropic-induced cardiovascular risk}

Several psychotropic medications used in the treatment and management of mental disorders are associated with increased cardiovascular-related health complications in people with serious mental illness. Psychotropic medications can both directly and indirectly increase the risk of developing cardio-metabolic effects (Walker et al, 2015; Kahl et al, 2018). Most, if not all antipsychotic medications, particularly second-generation antipsychotics, can increase the risk of developing obesity, weight gain, dyslipidaemia and diabetes. Subsequently, all these factors in turn are associated with increased cardiovascular related complications (Thornicroft, 2013; Tosh et al, 2014).

\section{Metabolic syndrome}

Metabolic syndrome, a cluster of conditions (high systolic blood pressure, large waist circumference, raised glycaemic levels, dyslipidaemia, insulin resistance, obesity) associated with increased risk of developing heart disease, diabetes and stroke is more prevalent in people with serious mental illness taking psychotropic medication (Mutsatsa, 2015). Sankaranarayan and Castle (2012) point out that metabolic syndrome is linked to a 2-3 times increased risk of cardiovascular health-related disability and mortality. Still, the prevalence of metabolic syndrome in those with serious mental illness is more than double the corresponding prevalence in people without mental illness; despite current debates and disagreements concerning the definition of metabolic syndrome and which of the clinical parameters to consider for diagnostic purposes, increasingly, the cardiometabolic problems are significantly more common in people with serious mental illness taking psychotropic medications (Reaven, 2006; Gale, 2008; Care Quality Commission, 2017; Ballon et al, 2018).

\section{Psychotropic-induced weight gain, glycaemic and lipid abnormalities}

Almost all psychotropic medications are associated with cardiometabolic adverse effects. However, as discussed, increased weight gain and obesity (risk factors for cardiovascular disease and diabetes) has been linked mainly to atypical antipsychotics, such as olanzapine and clozapine (Ballon et al, 2018), as well as low-potent typical antipsychotics such as chlorpromazine and levomepromazine (Tardy et al, 2014). A medium-risk incidence of weight gain has been reported in people taking other antipsychotics, such as risperidone, asenapine, quetiapine, haloperidol and fluphenazine; while a much lower risk of weight gain has been reported in people with serious mental illness taking aripiprazole and lurasidone (Meyer et al, 2015). In a randomised controlled trial of healthy volunteers treated with olanzapine, iloperidone or placebo for 28 days, Ballon et al (2018) found that gains in body weight and adipose tissue (a specialised form of connective tissue that acts to support, connect, or protect organs) mass in olanzapine-treated subjects were associated with increased caloric intake, which unsurprisingly, was further associated with development of dyslipidaemia (hypertriglyceridaemia and high total/ lowdensity lipoprotein cholesterol).

Unrelated to antipsychotic-induced weight and fat mass effects, olanzapine has been associated with early development of changes in glycaemic control and metabolism, suggesting that olanzapine, and possibly clozapine, may impair the activity of pancreatic insulin-secreting beta cells, even without the effects of weight increase (Simpson et al, 2012). Long-term treatment on psychotropic medications such 
cardiometabolic abnormalities, including increased peripheral insulin resistance, deteriorating pancreatic beta cell function, followed by glucose dysregulation and chronic hyperglycaemia. Evidence shows that dyslipidaemia in people with serious mental illness is higher than in people without mental illness; and particularly those taking antipsychotics are at an even greater risk of developing lipid or weight-related problems (Lester, 2012; World Health Organization, 2018).

The mechanism by which psychotropic medications, especially antipsychotics, induce weight and lipid abnormalities is not entirely understood and may be related or unrelated to medication doses. However, the common theory proposed relates to the pharmacodynamic and pharmacokinetic effects of psychotropic medication in the body. Evidence suggests that the therapeutic and adverse effects of psychotropic medication results from how the medications interact with serotonergic (5-HT2C and 5HT2A receptors), dopaminergic (D2 receptor) and, particularly histaminergic ( $\mathrm{H} 1$ receptor) antagonism (Cohen et al, 2013; Mangurian et al, 2016); 5-HT2C genetic polymorphism has also been implicated (Reynolds et al, 2002). All these neuro-circuit pathways play a role in the body from regulating feelings, emotions, thoughts, behaviours, conduct and energy regulation.

\section{Psychotropic-induced effects on the cardiovascular system}

The QT interval is the traditional time measure of ventricular contraction and relaxation represented on an electrocardiogram heart tracing. Congenital QT prolongation disorders are rare but are clinically associated with sudden death because of the potential risk of inducing cardiac arrhythmias, particularly a distinctive polymorphic ventricular tachycardia, commonly referred to as 'torsade de pointes' (TdP). QT prolongation can be affected by factors such as age, gender, activity, time of day and heart rate. To account for the effect of the heart rate, a more appropriate measure of QT interval with a correction for heart rate (QTC) is widely adopted. In people with serious mental illness taking psychotropic medication including antipsychotics and antidepressants, clinical symptoms including fainting, seizures and sudden death resulting from prolonged repolarisation have been reported.

Direct effects on the cardiovascular system by psychotropics have also been reported, including thrombotic events, bleeding, decrease or increase in blood pressure, arrhythmias, ventricular tachycardia and sudden death (Blankfield, 2012). Very severe antipsychotic-induced cardiovascular adverse effects, for example ventricular tachycardia and QT prolongation, have been linked to sudden death and were reported when antipsychotics were first used in clinical practice (Buckley and Sanders, 2000). Cardiovascular disease risk is increased in individuals with undiagnosed congenital QT interval prolongation taking antipsychotics, such as haloperidol and phenothiazines.

Several psychotropic medications (citalopram, haloperidol, risperidone, phenothiazines) can induce QTc prolongation; patients who are taking psychotropics and other medications associated with QTC prolongation (i.e. tricyclic antidepressants) are at increased risk of developing arrhythmias (Van Noord, 2009). Amisulpride is associated with QTc prolongation in overdose and can also cause torsade de pointes (Isbister et al, 2010); aripiprazole, olanzapine, paliperidone, quetiapine and risperidone are associated with risks of TdP (Haverkamp and Deuschle, 2006; Isbister et al, 2006) and clozapine has also been linked to QTc interval prolongation. Furthermore, clozapine has been found to increase the risks of venous thromboembolism and pulmonary embolism in people taking this medication (Stansby, 2010; Shulman et al, 2013). People with serious mental illness taking either haloperidol or chlorpromazine have a greater risk of developing QTc prolongation and torsade de pointes (Su et al, 2003). Atypical antipsychotics are associated with a lower risk of extrapyramidal side effects and death but have an 
elevated risk of stroke and even greater risk of patients developing serious ventricular arrhythmias (Crump et al, 2013; Farlow and Shamliyan, 2017).

Additionally, electrolyte abnormalities commonly reported in patients with serious mental illness include hypokalaemia, hypomagnesaemia and hypocalcaemia, and can increase the risk of developing arrhythmias (Taylor et al, 2015). Combination therapy involving diuretics can elevate this risk as diuretics have the marked potential to induce electrolyte abnormalities. Increased risk of developing arrhythmias and other cardiovascular related problems in serious mental illness is greater in individuals with cooccurring complex needs, for example drug and alcohol misuse. Individuals with liver cirrhosis either resulting from alcohol abuse and/or chronic hepatitis B or C are at increased risk of QTc interval prolongation and subsequent sudden death, mainly resulting from reduced hepatic function, thus affecting the liver's important role in drug metabolism (Mimidis et al, 2003).

Antipsychotic therapy can increase the risk of atrial arrhythmias, such as atrial fibrillation, which in turn is linked to stroke and heart failure. In a study by Chou et al (2017), antipsychotic use especially medications with greater binding affinity to muscarinic M2 receptors (haloperidol) were found to be associated with a higher incidence of atrial fibrillation. Patients with pre-existing cardiovascular disease and related conditions (e.g. coronary heart disease, diabetes, hypertension) have a higher risk of developing atrial fibrillation when taking antipsychotics. Manual blood pressure measurement is usually recommended for patients with atrial fibrillation because the pulse irregularity can inadvertently affect the automated blood pressure machines and evidence suggests that the output blood pressure readings from automated machines may not be accurate and reliable in patients with atrial fibrillation (HoganQuigley et al, 2012; Debbie et al, 2017).

Foley and Mackinnon (2014) conducted a systematic review of antipsychotic drug effects on human gene expression related to risk factors for cardiovascular disease. Antipsychotics were found to change the expression of several genes associated with cardiovascular health; unsurprisingly, some of the altered genes involved were those under the control of transcription factors that regulate lipid and fatty acid metabolism. Screening and monitoring for cardiovascular disease risk in serious mental illness should consider all modifiable and non-modifiable risk (genetic vulnerabilities, family history) and how both may interact and influence the course of clinical presentations.

Given the considerable clinical and economic burden of cardiovascular disease among people with serious mental illness, increasing focus is now on an integrated management model of physical and mental health care in practice. The challenges and/ or dilemmas in practice remain around balancing the risks of infrequent but serious iatrogenic adverse effects and clinical therapeutic benefits of psychotropics.

\section{Current practice}

Since the publication of the Chief Nursing Officer Review of Mental Health Nursing (Department of Health, 2006), over 250 best practice policy guidelines have been published providing mental health professionals with guidance and advice on managing physical and mental health needs in serious mental illness. Evidence-based guidelines bridge the policy-practice gap and help to draw the mental health professionals' attention to recognise and address known common cardiovascular disease risk factors (smoking, dyslipidaemia, hypertension, sedentary behaviour, obesity) in a timely fashion. The importance of providing timely interventions is further referenced in the NHS England education report, 'Making every contact count' (Varley and Murfin, 2018). Emphasis in this report and other health 
guidelines invites health professionals to consider, by identifying and targeting interventions to the most at-risk groups, including individuals with serious mental illness taking psychotropic medication, older adults and those with complex and/or co-occurring needs (Department of Health, 2016; National Institute for Health and Clinical Excellence (NICE), 2016). The impact of guidelines on practice and improvements on patient outcomes remains varied across clinical practice and particularly in mental health settings; while improvements in physical health care among serious mental illness have been made, there is still disparity between physical and mental health care provision across provider mental health settings (Disability Rights Commission, 2006; Baller et al, 2015; Mwebe, 2017).

\section{Lester Tool}

The simple guidance on screening for cardiometabolic risk in serious mental illness (Lester Cardiometabolic Tool, referred to as the Lester Tool, Lester et al, 2012) by the Royal Colleges, including those of General Practitioners and Psychiatrists, is an effective screening tool as an addition when care planning and conducting cardiometabolic screening and planning interventions. Using the tool as a track and trigger aid can help to identify health concerns early so that interventions can be targeted promptly (Shiers et al, 2014). The Lester Tool incorporates variable cardiovascular disease risk factors with parameters at which interventions should be offered when risk is identified and individualising care in cases where patients with known cardiovascular disease risk (arrythmias) are taking psychotropic medication. For instance, the Lester Tool can be used to screen and identify people with raised systolic blood pressure, glucose and lipid abnormalities; those who smoke, and patients who are overweight and/or are sedentary. An elevated blood pressure can result from antipsychotic medication or weight gain as a direct consequence of being obese/overweight. Feedback collected informally by one of the authors (HM) when delivering physical health training to mental health professionals from an NHS Trust suggests that clinical teams find the Lester Tool useful, but evidence of how often the tool is used across inpatient adult and older adult and community mental health services is still lacking; perhaps this disparity does affect the evaluation of effectiveness of this tool and others in clinical settings, and could explain low uptake and usage for the Tool by staff.

\section{Commissioning for Quality and Innovation}

Alongside the Lester Tool, provider services are incentivised and encouraged to use the existing national levers, for example national level physical health Commissioning for Quality and Innovations (NHS England, 2014b) to record and report on physical health care activities including screening, monitoring and uptake of interventions by people with serious mental illness. Doing so is a vital step to inform planning of resources (appropriating resources where needed) and tackling health disparities relating to access and provision of physical care activities in response to individuals' needs. In primary care, the Quality Outcomes Framework (QOF) is a voluntary incentive and reward programme for GP practices to improve the quality of care they provide to patients, it is aimed at helping to standardise improvements in the delivery of primary care. Quality Outcomes Framework screening checks offered to people with serious mental illness on practice registers include smoking, body mass index, blood pressure, glucose and cholesterol checks.

\section{$\underline{\text { QRISK3 }}$}

The QRISK3 calculator, an update of QRISK2, is a validated prediction assessment tool that is commonly used in primary care to estimate the 10-year risk of cardiovascular disease in women and men accounting for cardiovascular disease risk factors (Collins and Altman, 2012). The QRISK3, unlike QRISK2, incorporates antipsychotic medication and mental illness as risk factor variables to estimate cardiovascular disease risk - this is an important addition as people with serious mental illness are often exposed to psychotropic-induced cardiovascular effects unlike those without mental illness (NICE, 2015; 
Hippisley-Cox et al, 2017; Fe Barcones, 2018). The evidence of QRISK assessments for inpatients hospitalised within psychiatric settings for short or long stays (>6 months) remains low; it is unclear whether staff routinely use this tool alongside others to assess for cardiovascular disease risk in inpatients. Given that the QRISK tool has been validated and found to be clinically significant with very good performance and predictive power (Hippisley-Cox et al, 2017), the tool should be equally used across primary, secondary and community services to screen for cardiovascular disease risk alongside thorough assessment and examination of health needs in high risk individuals, namely patients who often miss or do not attend planned health reviews in general practice but may present to secondary care with periods of inpatient stays. In their Primrose Research study, Osborn et al (2015) derived two cardiovascular disease risk predication models specific to people with serious mental illness (the Primrose lipid model and the Primrose BMI model). These models include variables such as diagnosis, substance misuse, use of psychotropics and social deprivation score. The models can offer benefits for clinicians in understanding ways to manage and assess cardiovascular disease risk.

\section{National Early Warning Score 2 (NEWS 2)}

The National Early Warning Score (NEWS 2) developed by the Royal College of Physicians (2018) is an effective screening tool that can assist clinicians to identify patients at risk of physical deterioration (at risk of heart-respiratory related complications, sepsis, neurological deficits). The tool can be used to monitor changes in the physiological status of the patient, for instance falling or rising pulse rate or blood pressure, loss of consciousness, pyrexia, circulatory and respiratory changes. The tool is equally useful in chronic disease monitoring and symptoms arising from adverse effects from drug interactions and/or as a result of acute ill health.

\section{Behavioural change initiatives alongside routine physical health monitoring}

Behaviour change interventions using psychodynamic approaches, such as motivational interviewing, are useful and can be adopted in care planning activities and interventions to explore the individual's preparedness and attitude to changing unhealthy lifestyle behaviours. Such interventions should include but not limited to prompt physical health assessment (ask about medical history, family history, health beliefs and attitudes to lifestyle modification) and physical examinations for patients - blood pressure, pulse, weight monitoring, girth measures, and blood profile investigations including glucose checks and lipid profile to screen for abnormal cholesterol levels.

High blood cholesterol can cause fatty deposits (atherosclerosis) in blood vessels; these fatty deposits can make it difficult for enough blood to flow through the arteries. Being physically active for 30 minutes daily (including brisk walking and/ or running) can help to address weight gain and lipid abnormalities in individuals with known risk. In people with serious mental illness, especially those exposed to psychotropic medication, unhealthy blood fats (cholesterol and triglyceride levels), underactive thyroid gland (hypothyroid), type 2 diabetes, kidney problems, liver problems, high alcohol intake should be investigated and appropriate interventions offered.

\section{A summary of recommendations for practice}

- Obtain baseline personal and family history of diabetes, obesity, dyslipidaemia, hypertension and cardiovascular disease

- Monitor weight/body mass index/waist circumference before initiation of treatment, at 3 months and annually after 
- Monitor blood glucose (Fasting glucose/HbA1c) before initiation of treatment, at 3 months and annually after

- Monitor blood lipids (total cholesterol, non-high-density lipoprotein, high-density lipoprotein, triglycerides) before initiation of treatment, at 3 months and then annually

- Cholesterol levels naturally tend to increase with age, older adults aged 65 years and over taking psychotropic medications are particularly at greater risk of cardiovascular related complications (e.g. angina, heart attacks, vascular disease, stroke). Patients over 40 years, especially those with other known risk factors should be advised to access NHS health checks via general practice, high street pharmacies or from a secondary care team

- Advise and refer smokers with mental illness wanting to stop or cut down, encourage smokers to access NHS stop smoking services (accessible via inpatient wards, community health centres, high street pharmacies, and general practice)

- Health checks for serious mental illness patients should be offered at least every 6-12 months and should include electrocardiogram monitoring (before and after starting medication), dental and eye checks, enquiries about sleep regimes, offering advice on healthy eating, screening for psychosocial stressors as well as substance misuse

- Regular physical examinations for blood pressure, pulse, temperature, and respiration should be offered at least once a month in people with serious mental illness taking psychotropic medication

- Medication reviews should be offered regularly to assess for medication-induced adverse effects following the start of new medication, dose increases and where polypharmacy and high dose antipsychotic treatment are used

- Always discuss the results of any monitoring with the patient and jointly agree on an action plan.

\section{Conclusion}

Greater burden of morbidity and mortality in serious mental illness is often caused by natural causes including cardiovascular disease, diabetes and other medical conditions. Adverse effects from psychotropic interventions in serious mental illness further contribute to this burden, as well as the direct impact of suffering and living with a mental illness on the individual's physical health. People with serious mental illness are less likely than the general population to be offered physical health checks and monitoring for lipid, glucose checks, blood pressure, pulse, weight measurements and others; and when identified, their needs are not always promptly met.

All health professionals under various health and social care providers must collaboratively work together to tackle the high physical health burden in serious mental illness. This is particularly true for mental health professionals as the largest frontline staff group working in community, primary care and inpatient settings, and are aptly placed to make every contact count to offer the necessary physical health interventions including screening, monitoring and timely management of physical health needs in people with serious mental illness.

\section{References}

American Diabetes Association. Economic costs of diabetes in the US in 2012. Diabetes Care. 2013;36(4):1033-1046. https://doi.org/10.2337/dc12-2625 
Baller JB, McGinty EE, Azrin ST, Juliano-Bult D, Daumit GL. Screening for cardiovascular risk factors in adults with serious mental illness: a review of the evidence. BMC Psychiatry. 2015;15:55. https://doi.org/10.1186/s12888-015-0416-y

Ballon JS, Pajvani UB, Mayer LE et al. Pathophysiology of drug induced weight and metabolic effects: findings from an RCT in healthy volunteers treated with olanzapine, iloperidone, or placebo. J Psychopharmacol. 2018;32(5):533-540. https://doi.org/10.1177/0269881118754708

Bhatnagar P, Wickramasinghe K, Williams J, Rayner M, Townsend N. The epidemiology of cardiovascular disease in the UK 2014. Heart. 2015;101(15):1182-1189. https://doi.org/10.1136/heartjnl-2015-307516

Blankfield RP. Blood pressure, fluid retention and the cardiovascular risk of drugs. Future Cardiol. 2012;8(4):489-493. https://doi.org/10.2217/fca.12.39

British Heart Foundation. CVDT statistics-BHF UK factsheet. 2018. www.bhf.org.uk/research/heartstatistics (accessed 15 May 2019)

Buckley NA, Sanders P. Cardiovascular adverse effects of antipsychotic drugs. Drug Saf. 2000;23(3):215228. https://doi.org/10.2165/00002018-200023030-00004

Care Quality Commission. Brief guide: physical healthcare in mental health settings. London: CQC; 2017

Chou RH, Lo LW, Liou YJ et al. Antipsychotic treatment is associated with risk of atrial fibrillation: a nationwide nested case-control study. International Journal of Cardiology. 2017; 227:134-140. https://doi.org/10.1016/i.ijcard.2016.11.185

Cohen DL, Townsend RR. Blood pressure in patients with atrial fibrillation: part 1-measurement. J Clin Hypertens. 2017;19(1):98-99. https://doi.org/10.1111/jch.12905

Cohen T, Sundaresh S, Levine F. Antipsychotics activate the TGF $\beta$ pathway effector SMAD3. Mol Psychiatry. 2013;18(3):347-357. https://doi.org/10.1038/mp.2011.186

Collins GS, Altman DG. Predicting the 10-year risk of cardiovascular disease in the United Kingdom: independent and external validation of an updated version of QRISK2. BMJ. 2012;344:e4181. https://doi.org/10.1136/bmj.e4181

Copeland LA, Zeber JE, Valenstein M, Blow FC. Racial disparity in the use of atypical antipsychotic medications among veterans. American Journal of Psychiatry. 2003;160(10):1817-1822. https://doi.org/10.1176/appi.ajp.160.10.1817

Correll CU, Solmi M, Veronese $\mathrm{N}$ et al. Prevalence, incidence and mortality from cardiovascular disease in patients with pooled and specific severe mental illness: a large-scale meta-analysis of 3,211,768 patients and 113,383,368 controls. World Psychiatry. 2017;16(2):163-180. https://doi.org/10.1002/wps.20420

Crump C, Winkleby MA, Sundquist K, Sundquist J. Comorbidities and mortality in persons with schizophrenia: a Swedish National Cohort Study. American Journal of Psychiatry. 2013;170(3):324-333. https://doi.org/10.1176/appi.ajp.2012.12050599

Das-Munshi J, Bhugra D, Crawford MJ. Ethnic minority inequalities in access to treatments for schizophrenia and schizoaffective disorders: findings from a nationally representative cross-sectional study. BMC Med. 2018;16(1):55. https://doi.org/10.1186/s12916-018-1035-5 
Disability Rights Commission. Equal treatment: closing the gap. 2006. https://disabilitystudies.leeds.ac.uk/wp-content/uploads/sites/40/library/DRC-Health-FI-main.pdf (accessed 11 June 2019)

Department of Health. Choosing health: supporting the physical health needs of people with severe mental health illness. Commissioning framework. London: DH; 2006

Department of Health. No health without mental health: a cross-government mental health outcomes strategy for people of all ages. London: $\mathrm{DH} ; 2016$

Farlow MR, Shamliyan TA. Benefits and harms of atypical antipsychotics for agitation in adults with dementia. European Neuropsychopharmacology. 2017;27(3):217-231. https://doi.org/10.1016/j.euroneuro.2017.01.002

Fe Barcones M, MacDowell KS, García-Bueno B et al. Cardiovascular risk in early psychosis: relationship with inflammation and clinical features 6 months after diagnosis. Int J Neuropsychopharmacol. 2018;21(5):410-422. https://doi.org/10.1093/ijnp/pyx110

Foley DL, Mackinnon A. A systematic review of antipsychotic drug effects on human gene expression related to risk factors for cardiovascular disease. Pharmacogenomics J. 2014;14(5):446-451. https://doi.org/10.1038/tpj.2014.8

Gale EA. Should we dump the metabolic syndrome? Yes. BMJ. 2008;336(7645):640. https://doi.org/10.1136/bmj.39477.500197.AD

Hardy S, White J. Why are people with serious mental illness still not getting their physical health checked? Ment Health Nurs. 2013;33(1):14-18

Haverkamp W, Deuschle M. Lengthening of QT interval by antipsychotic drugs. Nervenarzt. 2006;77(3):276-284. https://doi.org/10.1007/s00115-005-1966-x

Health Education England. Raising the bar: shape of caring: a review of the future education and training of registered nurses and care assistants. London: HEE; 2016

Heart UK. Key facts \& figures. 2018. https://heartuk.org.uk/press/press-kit/key-facts-figures (accessed 30 May 2019)

Hippisley-Cox J, Coupland C, Brindle P. Development and validation of QRISK3 risk prediction algorithms to estimate future risk of cardiovascular disease: prospective cohort study. BMJ. 2017;357:j2099. https://doi.org/10.1136/bmi.j2099

Hogan-Quigley B, Palm ML, Bickley LS. Bates' nursing guide to physical examination and history taking. Philadelphia PA: Lippincott, Williams and Wilkins; 2012

Holt R. Cardiovascular disease and diabetes in people with severe mental illness: causes, consequences and pragmatic management. PCCJ. 2012;5(2):81-85

Isbister GK, Balit CR, Macleod D, Duffull SB. Amisulpride overdose is frequently associated with QT prolongation and torsades de pointes. Journal of Clinical Psychopharmacology. 2010;30(4):391-395. https://doi.org/10.1097/JCP.0b013e3181e5c14c 
Isbister GK, Murray L, John S et al. Amisulpride deliberate self-poisoning causing severe cardiac toxicity including QT prolongation and torsades de pointes. Med J Aust. 2006;184(7):354-356. https://doi.org/10.5694/j.1326-5377.2006.tb00272.x

Kahl GK, Westhoff-Bleck M, Kruger THC. Effects of psychopharmacological treatment with antipsychotic drugs on the vascular system. Vascular Pharmacology. 2018;100:20-25. https://doi.org/10.1016/j.vph.2017.09.001

Laursen TM, Munk-Olsen T, Vestergaard M. Life expectancy and cardiovascular mortality in persons with schizophrenia. Current Opinion in Psychiatry. 2012;25(2):83-88. https://doi.org/10.1097/YCO.0b013e32835035ca

Lester $\mathrm{H}$, Shiers D, Rafi I. Positive cardiometabolic health resource: an intervention framework for patients with psychosis on antipsychotic medication. London: Royal College of Psychiatrists; 2012

Mangurian C, Newcomer JW, Modlin C, Schillinger D. Diabetes and cardiovascular care among people with severe mental illness: a literature review. J Gen Intern Med. 2016;31(9):1083-1091. https://doi.org/10.1007/s11606-016-3712-4

Meyer JM, Mao Y, Pikalov A, Cucchiaro J, Loebel A. Weight change during long-term treatment with lurasidone: pooled analysis of studies in patients with schizophrenia. Int Clin Psychopharmacol. 2015;30(6):342-350. https://doi.org/10.1097/YIC.0000000000000091

Mimidis K, Papadopoulos V, Thomopoulos $\mathrm{K}$ et al. Prolongation of the QTc interval in patients with cirrhosis. Ann Gastroenterol. 2003;16(2):155-158

Mutsatsa S. Physical healthcare and promotion in mental health nursing. London: Sage; 2015

Mwebe H. Physical health monitoring in mental health settings: a study exploring mental health nurses' views of their role. J Clin Nurs. 2017;26(19-20):3067-3078. https://doi.org/10.1111/jocn.13653

Mwebe H. Serious mental illness and smoking cessation. Br J Ment Health Nurs. 2018;7(1):39-46. https://doi.org/10.12968/bjmh.2018.7.1.39

Nash M. Diagnostic overshadowing: a potential barrier to physical health care for mental health service users. Ment Health Pract. 2013;17(4):22-26. https://doi.org/10.7748/mhp2013.12.17.4.22.e862

National Institute for Health Clinical Excellence. Indicators for the NICE menu for the QOF: serious mental illness [NM120]. 2015. http://bit.ly/2WCCBzM (accessed 11 June 2019)

National Institute for Health Clinical Excellence. CVD risk assessment and management in primary and secondary care. Clinical guideline [CG181]. 2016. https://www.nice.org.uk/guidance/cg181 (accessed 30 May 2019)

NHS Digital. Detentions under the Mental Health Act. 2018. Ethnicity facts and figure: health. http://bit.ly/2KMOvv3 (accessed 11 June 2019)

NHS England. NHS five year forward view. 2014a. https://www.england.nhs.uk/wpcontent/uploads/2014/10/5yfv-web.pdf (accessed 31 May 2019)

NHS England. Commissioning for quality and innovation (CQUIN): 2014/15 guidance. Leeds: NHS England; 2014b 
Osborn DP, Hardoon S, Omar RZ et al. Cardiovascular risk prediction models for people with severe mental illness: results from the prediction and management of cardiovascular risk in people with severe mental illnesses (PRIMROSE) research program. JAMA Psychiatry. 2015;72(2):143-151. https://doi.org/10.1001/jamapsychiatry.2014.2133

Pilgrim D. Key concepts in mental health. 4th edn. London: Sage Publications; 2017

Reaven GM. The metabolic syndrome: is this diagnosis necessary? Am J Clin Nutr. 2006;83(6):12371247. https://doi.org/10.1093/ajcn/83.6.1237

Reynolds GP, Zhang ZJ, Zhang XB. Association of antipsychotic drug induced weight gain with a 5-HT2C receptor polymorphism. Lancet. 2002;359(9323):2086-2087. https://doi.org/10.1016/S0140$\underline{6736(02) 08913-4}$

Robson D, Haddad M, Gray R, Gournay K. Mental health nursing and physical health care: a crosssectional study of nurses' attitudes, practice, and perceived training needs for the physical health care of people with severe mental illness. Int J Ment Health Nurs. 2013;22(5):409-417. https://doi.org/10.1111/j.1447-0349.2012.00883.x

Royal College of Physicians. National Early Warning Score (NEWS) 2. Standardising the assessment of acute-illness severity in the NHS. 2018. http://bit.ly/2R3VroC (accessed 3 June 2019)

Sankaranarayan A, Castle D. Monitoring and managing metabolic syndrome in person with mental illness: the case for. AP J Psychol Med. 2012;13(1):9-13

Shiers DE, Rafi I, Cooper SJ, Holt RG. Positive cardiometabolic health resource: an intervention framework for patients with psychosis and schizophrenia. 2014 update (with acknowledgement to the late Helen Lester for her contribution to the original 2012 version). London: Royal College of Psychiatrists; 2014

Shulman M, Njoku JI, Manu P. Thrombotic complications of treatment with antipsychotic drugs. Minerva Med. 2013;104(2):175-184

Silverwood V, Chew-Graham C, and Shiers, D. (2019) Improving the physical health of people with severe mental illness. InnovAiT; Vol 12(4);203-210

Simpson N, Maffei A, Freeby $M$ et al. Dopamine-mediated autocrine inhibitory circuit regulating human insulin secretion in vitro. Mol Endocrinol. 2012;26(10):1757-1772. https://doi.org/10.1210/me.2012$\underline{1101}$

Stansby G, Noble S, Howes O. The importance of venous thromboembolism: a physical consequence of psychiatric treatments. Int J Clin Pract. 2010;64(8):1005-1006. https://doi.org/10.1111/j.1742$\underline{1241.2010 .02435 . x}$

Su KP, Shen WW, Chuang CL, Chen KP, Chen CC. A pilot cross-over design study on QTc interval prolongation associated with sulpiride and haloperidol. Schizophr Res. 2003;59(1):93-94. https://doi.org/10.1016/S0920-9964(01)00336-X

Tardy M, Dold M, Engel RR, Leucht S. Trifluoperazine versus low-potency first-generation antipsychotic drugs for schizophrenia. Cochrane Database of Systematic Reviews. 2014;(7):CD009396. https://doi.org/10.1002/14651858.CD009396.pub2 
Thornicroft G. Premature death among people with mental illness. BMJ. 2013;346:f2969. https://doi.org/10.1136/bmj.f2969

Tosh G, Clifton AV, Xia J, White MM. General physical health advice for people with serious mental illness. Cochrane Database of Systematic Reviews. 2014;(3):CD008567. https://doi.org/10.1002/14651858.CD008567.pub3

Van Noord C, Straus SM, Sturkenboom MC et al. Psychotropic drugs associated with corrected QT interval prolongation. Journal of Clinical Psychopharmacology. 2009;29(1):9-15. https://doi.org/10.1097/JCP.0b013e318191c6a8

Varley E, Murfin M. An implementation guide and toolkit for making every contact count: using every opportunity to achieve health and wellbeing: East Midlands Health Trainer Hub. Leeds: Health Education England; 2018

Walker ER, McGee RE, Druss BG. Mortality in mental disorders and global disease burden implications: a systematic review and meta-analysis. JAMA Psychiatry. 2015;72(4):334-341. https://doi.org/10.1001/jamapsychiatry.2014.2502

World Health Organization. Information sheet: premature death among people with severe mental disorders. Fact Sheet. 2018. https://www.who.int/mental health/management/info sheet.pdf (accessed 31 May 2019) 